\title{
Subwavelength Grating Devices in Silicon Photonics
}

\author{
Junjia Wang ${ }^{1}$, Ivan Glesk ${ }^{2}$, and Lawrence R. Chen ${ }^{1 *}$ \\ ${ }^{1}$ Department of Electrical and Computer Engineering, McGill University \\ Montreal, QC H3A 0E9 Canada \\ ${ }^{2}$ Department of Electronic and Electrical Engineering, University of Strathclyde \\ Glasgow G1 1XW UK \\ *lawrence.chen@mcgill.ca
}

\begin{abstract}
Subwavelength grating (SWG) waveguides in silicon-on-insulator are emerging as an enabling technology for implementing compact, high-performance photonic integrated devices and circuits for signal processing and sensing applications. We provide an overview of our recent work on developing wavelength selective SWG waveguide filters based on Bragg gratings and ring resonators, as well as optical delay lines. These components increase the SWG waveguide component toolbox and can be used to realize more complex photonic integrated circuits with enhanced or new functionality.
\end{abstract}

Keywords: Subwavelength gratings, silicon photonics, integrated optics, Bragg gratings, ring resonators, optical delay lines

\section{Introduction}

There is a growing need to develop integrated components for optical sensing and to perform a variety of signal processing functions in broadband (optical and microwave photonics) communications. Over the past few years, the development of active and passive devices in silicon photonics has been the subject of intense research and a number of enabling technologies have been demonstrated [1].

While the principles of subwavelength grating (SWG) structures have been long known, it has only been in the past few years that they have attracted interest for developing integrated optical components [2,3]. Early research focused on crosswise operation where light propagates orthogonally to the subwavelength structure and various silicon-on-insulator (SOI)-based devices and applications were reported, most notably, efficient fiber-to-chip surface grating couplers [4,5].

Recently, it was shown that light can propagate lengthwise in the subwavelength structure and be guided, in the same way as in a conventional waveguide. This observation gave rise to so-called microphotonic SWG waveguides [6]. In theory, such waveguides are lossless whereas in practice, a loss as low as $2.1 \mathrm{~dB} / \mathrm{cm}$ was obtained for an SWG waveguide in SOI. SWG waveguides have enabled a whole new platform in silicon photonics and indeed, a variety of devices have been proposed and demonstrated, including low loss $(<1 \mathrm{~dB})$ SWG tapers for coupling efficiently SWG waveguide devices to conventional silicon strip waveguides; SWG directional couplers with over $40 \mathrm{~nm}$ bandwidth and compact multimode interference couplers [7]; SWG waveguide crossings with negligible losses and waveguide bends with a loss as low as $0.8 \mathrm{~dB}$ per $90^{\circ}$ bend [8,9]; mode transformers for butt coupling and mode conversion [10]; and polarization converters [11]. All of these devices, with the exception of the polarization converter, can be fabricated with a single etch process. Moreover, they are compatible with one another and can be used to create more complex integrated devices, subsystems, and optical links. However, one 
fundamental building block that is missing in the SWG waveguide component toolbox is a wavelength selective filter.

In this paper, we review recent work on developing wavelength selective SWG waveguide devices based on Bragg gratings (BGs) [12,13] and ring resonators $[12,14,15]$, as well as optical delay lines (ODLs) [16]. The SWG BG and ring resonator filters can be used to develop more advanced wavelength selective components, such as optical add-drop multiplexers. The SWG ODLs can be used to implement the waveguide array in arrayed waveguide gratings and can also have important applications in optical communications and microwave photonic systems, such as to provide synchronization for all-optical signal processing or broadband phase-shifting for phase array antennas and beamforming.

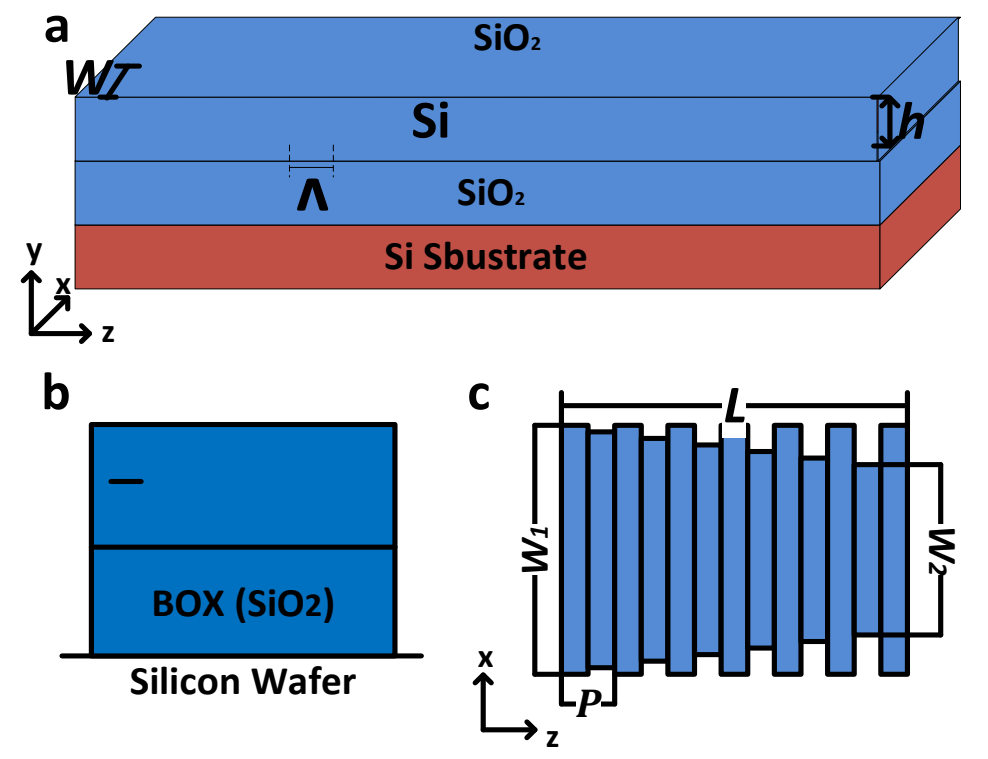

Fig. 1. (a) SWG waveguide implemented in SOI, (b) cross-section of the waveguide, and (c) top view of the SWG taper.

\section{Background}

SWGs are grating structures with a period $\Lambda$ that is sufficiently small compared to the wavelength of light. They are formed by a periodic arrangement (with period $\Lambda$ ) of high refractive index material with a thickness $a$ implanted into a low refractive index material (the duty cycle of the SWG is defined as $D=a / \Lambda$ ). To create an SWG waveguide, finite transverse dimensions (e.g., a width $W$ and height $h$ ) are applied to the material of high refractive index. Light propagation in the direction along the periodic refractive index arrangement (i.e., lengthwise operation) is similar to electron propagation in a periodic potential and can be described using Bloch waves. The carrier frequency of the light signal propagating in the SWG determines the operating regime. At low frequencies, the propagation constant $\left(k_{\mathrm{B}}\right)$ increases with frequency in a similar manner to a conventional waveguide, i.e., the subwavelength regime. Once the frequency increases to the photonic bandgap, Bragg reflection occurs and light is reflected back, i.e., the Bragg reflection regime. Above the first bandgap is the radiation regime where the Bloch mode becomes leaky and light is scattered out of the waveguide, i.e., the radiation regime. We are particularly interested in the subwavelength regime where the propagating light perceives the periodic structure as an effective medium. In other words, the SWG waveguide can be modeled as a 
conventional strip waveguide having the same transverse dimensions and a uniform refractive (effective) index along the direction of propagation. The effective index of the waveguide depends on the duty cycle $[6,17,18]$.

SWG waveguides in SOI can be realized by a periodic arrangement of silicon and silica as shown schematically in Fig. 1(a). For fabrication processes available from typical silicon photonic multi-project wafer runs, $h=220 \mathrm{~nm}$ and the silicon layer sits on top of a $3 \mu \mathrm{m}$ buried-oxide (BOX) layer; it is covered by a $2 \mu \mathrm{m}$ cladding that is index-matched to the BOX layer, see Fig. 1(b). The SWG waveguides considered throughout this paper also include an input and an output SWG taper. The taper serves to convert a conventional waveguide mode propagating in a silicon strip waveguide into a Bloch mode that propagates in the SWG waveguide. The taper is based on a linearly chirped waveguide grating implemented with a uniform period $P$ and where the waveguide width is narrowed linearly from $W_{1}$ to $W_{2}$ over a length $L_{\text {taper }}$, see Fig. 1(c) [8]. Figure 2 shows the E-field propagating in a strip waveguide and an SWG waveguide in SOI calculated using a 2.5D FDTD simulator (Lumerical ${ }^{\mathrm{TM}}$ ). The waveguides have the same cross-section of $W=500 \mathrm{~nm} \times h=220 \mathrm{~nm}$, and the SWG waveguide has a period $\Lambda=300 \mathrm{~nm}$ and $D=50 \%$. We can clearly observe the difference between the TE-like mode propagating in the strip waveguide and the Bloch mode propagating in the SWG waveguide: for the latter, the intensity is high not only in the center of the waveguide, but also at the edges.
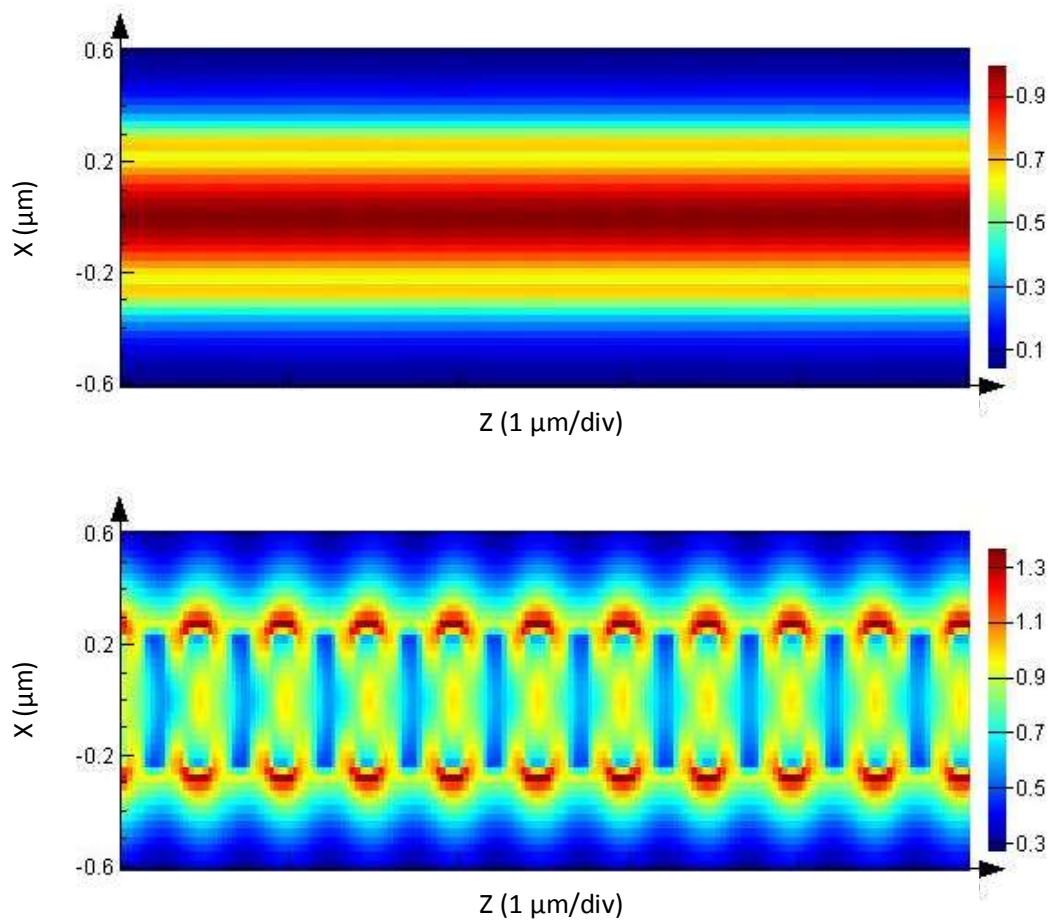

Fig. 2. Comparing the $E$-field profile for a TE-like mode propagating in a strip waveguide and a Bloch mode propagating in an SWG waveguide in SOI (the waveguides have the same cross-section).

\section{SWG BGs}

BGs are key structures for developing wavelength selective devices. Fiber Bragg gratings (FBGs) are a mature technology and have been broadly deployed in optical communications and sensing applications [19]. Integrated waveguide BGs bring the 
functionality of FBGs to the level of photonic integrated circuits. They can be realized by inducing periodic variations in the refractive index along the direction of propagation as well as physical corrugations or variations in the waveguide geometry. Waveguide BGs have been produced in a variety of material platforms, including silica and silicon-on-insulator [20-23]. Moreover, more complex structures have been designed to realize various signal processing and filtering functions [24-28].
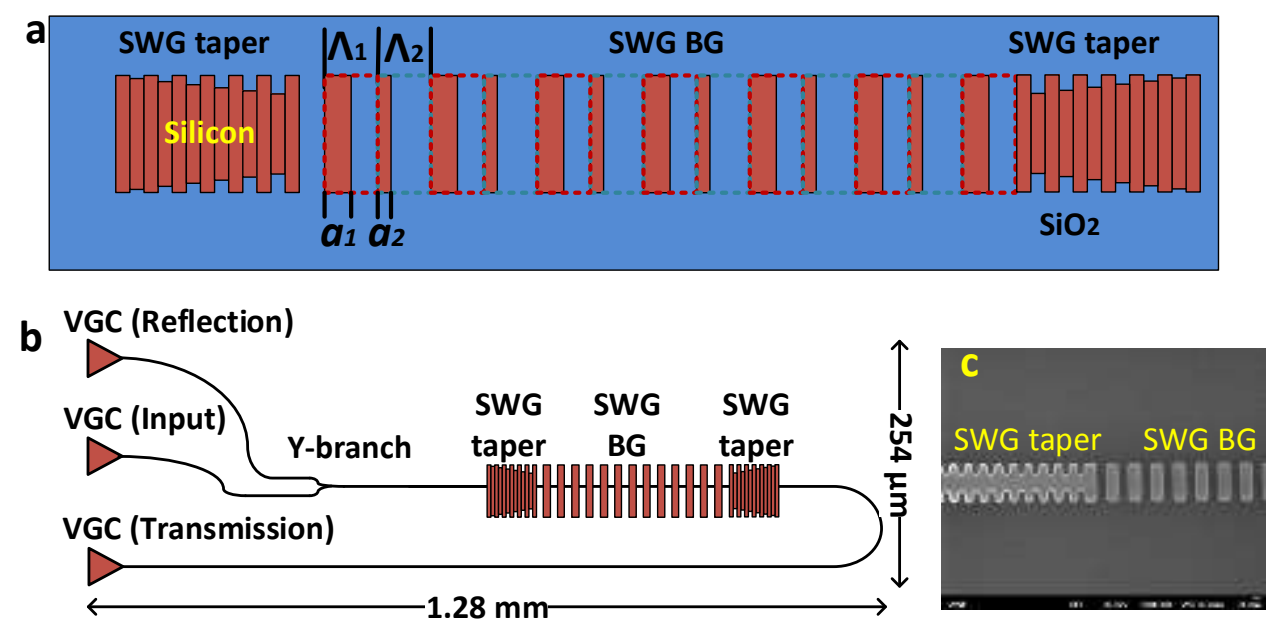

Fig. 3. (a) schematic of BG formed by interleaving two SWG waveguides of different duty cycles, (b) device layout for experimental demonstration, and (c) SEM of the fabricated SWG BG prior to oxide cladding deposition. VGC: vertical grating coupler.

As mentioned in Section 2, the effective/group index of an SWG waveguide depends on the duty cycle. By 'interleaving' two SWG waveguides with different duty cycles, we can create a periodic variation in effective index along the direction of propagation and hence realize a Bragg structure, i.e., and an SWG BG. Figure 3(a) shows a schematic of an SWG BG in which two SWG waveguides with different duty cycles $D_{1}=\left(a_{1} / \Lambda_{1}\right)$ and $D_{2}=\left(a_{2} / \Lambda_{2}\right)$ are interleaved; the period of the SWG BG is $\Lambda_{1}$ $+\Lambda_{2}$.

To verify the principle of operation, we calculate the spectral responses of different SWG BGs using a 2.5D FDTD simulator. The SWG waveguide has a crosssection of $W=450 \mathrm{~nm}$ and $h=220 \mathrm{~nm}$; the taper is formed by a linearly chirped grating with a period $P=200 \mathrm{~nm}$ and the waveguide width is narrowed from $W_{l}$ (= $W)=450 \mathrm{~nm}$ to $W_{2}=200 \mathrm{~nm}$ over a length $L_{\text {taper }}=15 \mu \mathrm{m}$. Figure 4 shows the transmission and reflection spectra of SWG BGs with $D_{1}=50 \%$ and different values of $D_{2}$ with $\Lambda_{1}=\Lambda_{2}=300 \mathrm{~nm}$ and 2,000 grating periods (corresponding to an SWG BG length of $1.2 \mathrm{~mm}$ ). Note that increasing $D_{2}$ causes the Bragg wavelength to shift to longer values. While 2.5D FDTD simulations are not as robust for determining exact quantitative results as their full 3D FDTD counterparts or the Fourier modal method [29], they are less computationally intensive and can nonetheless be used to understand general device characteristics. 

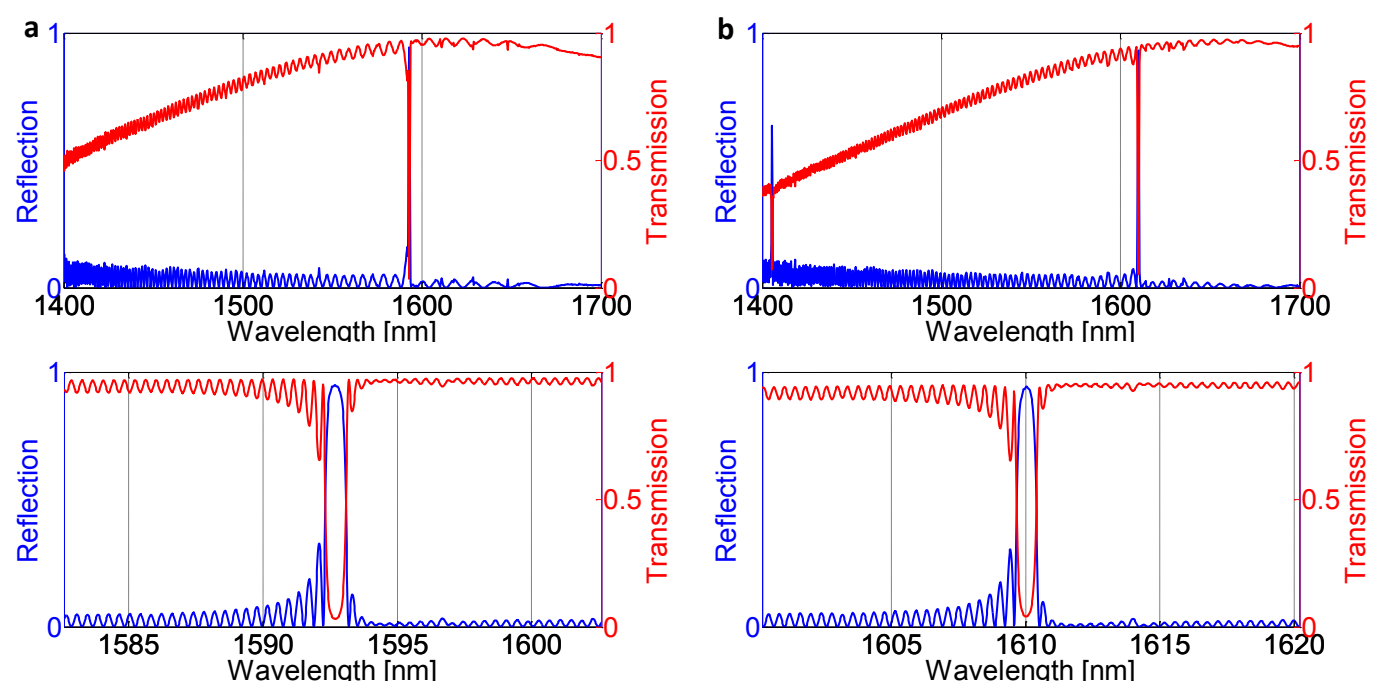

Fig. 4. Simulated transmission and reflection spectra of SWG BGs with (a) $D_{1}=50 \%$ and $D_{2}=46.67 \%$ and (b) $D_{1}=50 \%$ and $D_{2}=53.33 \%$. The corresponding zoomed responses are shown below.

As an experimental demonstration, we consider SWG waveguides with a cross section of $W=500 \mathrm{~nm}$ and $h=220 \mathrm{~nm}$; the taper uses a period of $P=200 \mathrm{~nm}$ and the waveguide width is reduced from $500 \mathrm{~nm}$ to $200 \mathrm{~nm}$ over a length $L_{\text {taper }}=15 \mu \mathrm{m}$. From our measurements of the transmission window for simple SWG waveguides, we use $\Lambda_{1}=\Lambda_{2}=280 \mathrm{~nm}$ for operation in the C-band. For the SWG BG, we consider 2,000 periods corresponding to a total length of $1.12 \mathrm{~mm}$. We use a compact $\mathrm{Y}$ branch [30] to extract the reflection response of the SWG BGs. Vertical grating couplers (VGCs) optimized for TE transmission serve to couple light into and out of the device [31]. The VGCs are separated by $127 \mu \mathrm{m}$ and a fiber ribbon array is used for testing. The device occupies a total footprint of $1.18 \mathrm{~mm} \times 254 \mu \mathrm{m}$. All the devices are fabricated using ebeam lithography and a single full etch. Figures $3(\mathrm{~b})$ and 3(c) show the device layout and an SEM image of a SWG BG before deposition of the top oxide, respectively.

All spectral responses are measured with a tunable laser scanned in steps of $10 \mathrm{pm}$ and an optical power meter. The total fiber-to-fiber loss is $\sim 15 \mathrm{~dB}$. Figure 5 compares the transmission response of an SWG BG with $D_{1}=50 \%$ and $D_{2}=48 \%$ and that of a simple SWG waveguide. The SWG BG exhibits a clear rejection peak at a resonant wavelength of $1546.8 \mathrm{~nm}$ and the transmission loss is $-12 \mathrm{~dB}$ corresponding to a peak reflectivity of $90.4 \%$ with a $3 \mathrm{~dB}$ bandwidth of $0.5 \mathrm{~nm}$. 


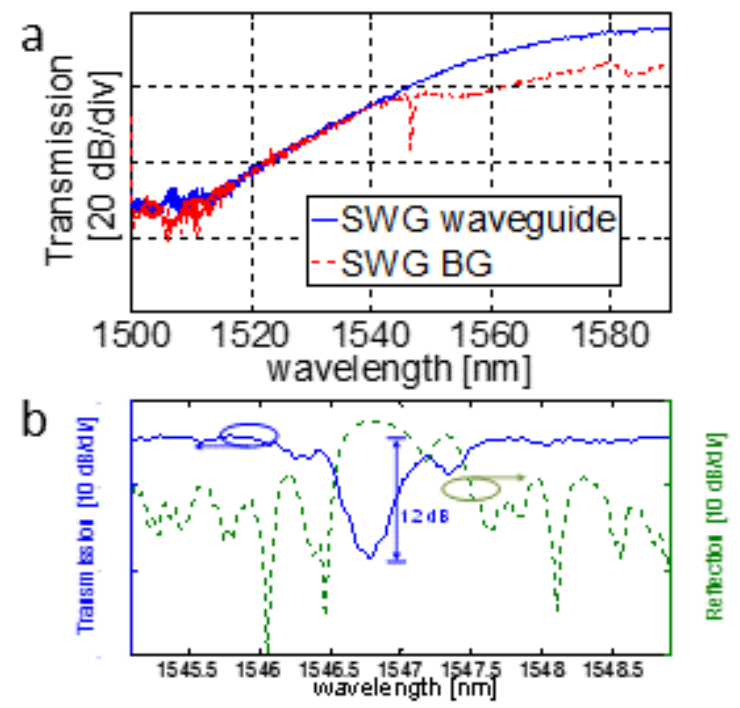

Fig. 5. (a) Measured transmissions spectrum of SWG BG and simple SWG waveguide (see text for parameters). (b) Zoom of the transmission and reflection responses about the resonant peak at $1546.8 \mathrm{~nm}$.

Figure 6 compares the reflection spectrum of the above SWG BG to one with $D_{1}$ $=50 \%$ and $D_{2}=44 \%$. As predicted by the simulations, a higher value of $D_{2}$ results in a longer Bragg wavelength. The second SWG BG with a smaller value of $D_{2}$ is centered at $1533.2 \mathrm{~nm}$ and has a peak reflectivity of $94.4 \%$ and $3 \mathrm{~dB}$ bandwidth of 0.5 $\mathrm{nm}$. Note that the reflection responses are not ideal (they do not have the exact same features as a conventional uniform BG) and exhibit ripples, the appearance of a resonance splitting, and broadband reflection. We believe that these are due in part to (1) spurious reflections between the SOI strip waveguide and SWG waveguide and (2) fabrication errors, including roughness arising from the etching process. The spectral responses can be improved using an optimized tapered waveguide design (to increase coupling and reduce mismatch between the strip and SWG waveguides) as well as better fabrication/processing.
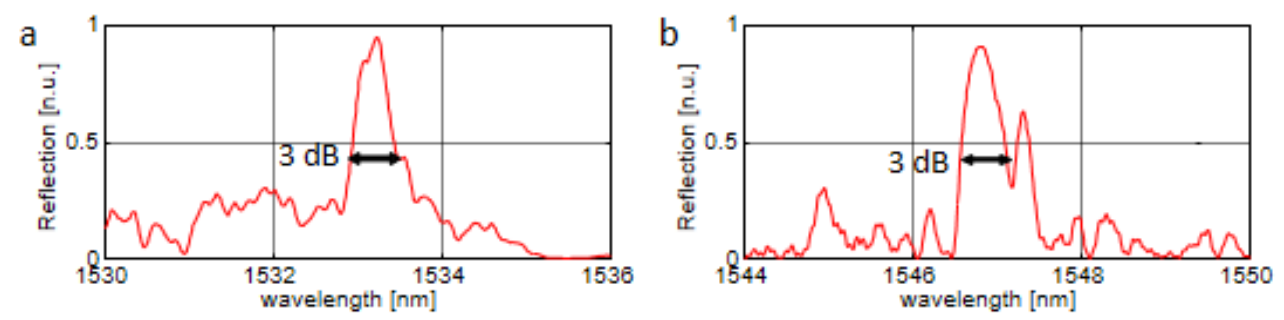

Fig. 6. Reflection response of SWG BGs: (a) $D_{1}=50 \%$ and $D_{2}=44 \%$ and (b) $D_{1}=$ $50 \%$ and $D_{2}=48 \%$.

\section{SWG ring and racetrack resonator filters}

In addition to BGs, ring resonators are also very effective wavelength selective devices. Indeed, many ring resonator-based devices in SOI have been demonstrated, including filters [32], modulators [33], and slow light/tunable delay line structures $[34,35]$. In this section, we investigate the feasibility of developing SWG-based ring resonators. 

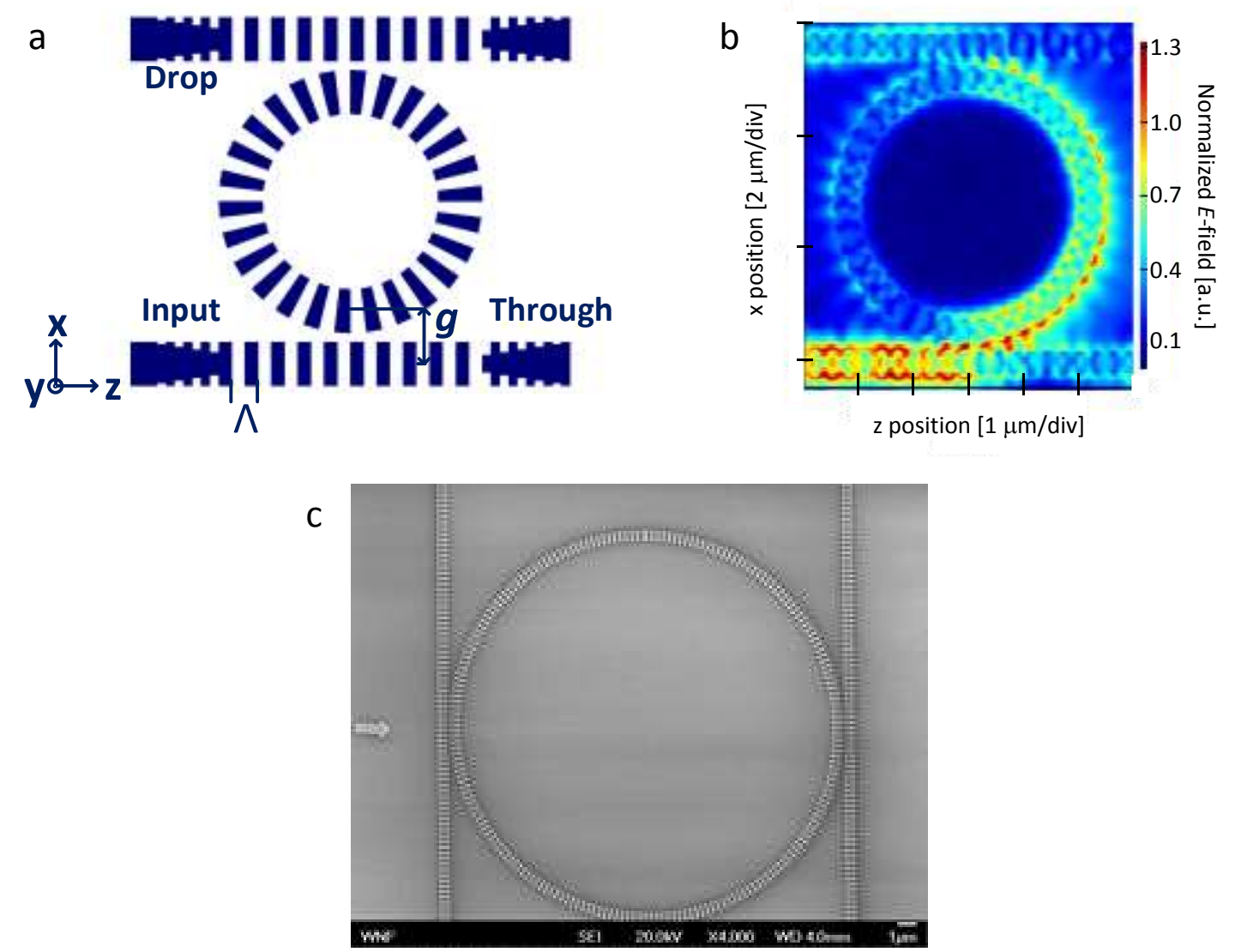

Fig. 7. (a) Schematic or SWG ring resonator, (b) top view of $E$-field propagating in the SWG ring resonator simulated using 2.5D FDTD, and (c) SEM of a fabricated SWG ring resonator before top oxide cladding deposition.

We first consider a simple add-drop configuration comprising two SWG bus waveguides and an SWG ring waveguide as illustrated in Fig. 7(a). For the simulations, we consider SWG waveguides with dimensions $W=450 \mathrm{~nm} \times h=220$ $\mathrm{nm}$, a period $\Lambda=300 \mathrm{~nm}$, and a duty cycle $D=50 \%$. Tapers similar to those described in Section 3 are used to couple light into and out of the SWG waveguides. We use directional coupling between the SWG bus and ring waveguides with a gap of $g=550 \mathrm{~nm}$ and the ring radius is $10 \mu \mathrm{m}$. In the SWG ring, the period is roughly 300 $\mathrm{nm}$ as well, but this depends on the position of the segments as the SWG ring is sliced by degrees. For instance, for an SWG ring with $10 \mu \mathrm{m}$ radius, two adjacent segments are separated by 0.015 radians according to the center, and the width of the segments is approximately equal to the length of the arc with 0.015 radians of central angle. Figure 7(b) shows a top view of the $E$-field propagating in the SWG ring resonator simulated using 2.5D FDTD. The SWG ring resonator operates similarly to a conventional ring resonator in that light coupled into and circulated in the cavity; the difference lies in the nature of light propagation in the SWG waveguides (note the Bloch modes in both the SWG bus and ring waveguides). 

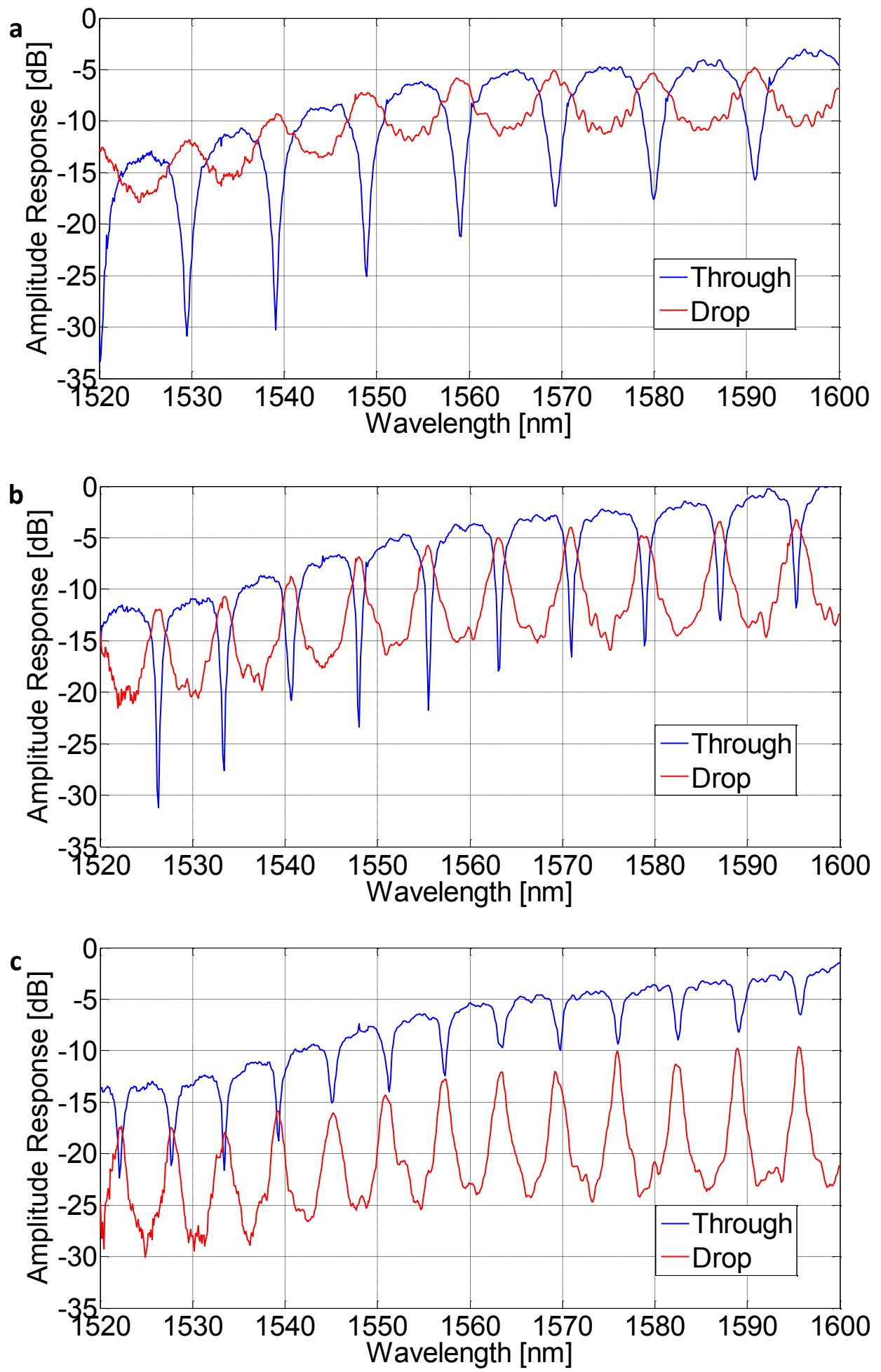

Fig. 8. Measured through and drop responses (10 pm resolution) of SWG ring resonators with a ring radius of (a) $15 \mu \mathrm{m}$, (b) $20 \mu \mathrm{m}$, and (c) $25 \mu \mathrm{m}$.

As an experimental demonstration, we fabricated ring resonators using ebeam beam lithography and a full etch with top oxide cladding. The parameters are similar to those from the simulations: the waveguide dimensions are $W=500 \mathrm{~nm} \times h=220$ $\mathrm{nm}$, the SWG grating period is $\Lambda=300 \mathrm{~nm}$, the duty cycle is $D=50 \%$, and $g=640$ 
nm. Tapers and VGCs are similar to those used to realize the BGs experimentally (see Section 3). All of the spectral measurements are normalized to the transmission response of the VGCs which are characterized separately via transmission measurements through a short length of strip waveguide. Figure 8 shows the measured through and drop responses for SWG ring resonators with different ring radii and the characteristics are summarized in Table 1. As expected, larger rings result in smaller values of free spectral range (FSR). The $Q$-factor (defined as $\lambda_{0} / \Delta \lambda$ where $\lambda_{0}$ is the central wavelength of a resonance and $\Delta \lambda$ is the corresponding $3 \mathrm{~dB}$ bandwidth) and extinction ratio (ER) of the resonance peaks depend on the total resonator losses [36] (in the discussion that follows and the results presented, the ER refers to that measured at the through response). The duty cycle $D$ of the SWG waveguide and the bend radius are instrumental in determining the total resonator losses, and hence, the $Q$-factor and ER. In [15], a detailed characterization of SWG ring resonators was performed with experimental measurements being in good agreement with full 3D FDTD simulations. The analysis showed that rings based on SWG waveguides with a smaller duty cycle have a higher $Q$-factor and ER for larger radii (e.g., $\geq 30 \mu \mathrm{m}$ ). This is due to the smaller loss associated with a smaller duty cycle (e.g., for $D=70 \%$ compared to $80 \%$ ). On the other hand, rings based on SWG waveguides with a larger duty cycle have a higher $Q$-factor and ER for small radii (e.g., $10 \mu \mathrm{m}$ ). This is since SWG waveguides with lower duty cycle and small bend radii have higher mode mismatch (and hence higher total bending loss). With our devices, we obtained $Q$-factors ranging from several hundred to $\sim 1,200$ with a top oxide cladding; in [15] $Q$-factors from 1,000 to 6,000 with an air cladding or $\sim 4,000$ with a fluidic cladding were achieved. Reducing total bending and scattering losses further (e.g., via improved fabrication/processing) will be necessary to enhance the $Q$ factor and/or ER of SWG ring resonators to make them comparable with ring resonators based on conventional SOI strip waveguides.

Table 1 Parameters obtained from experiment measurements

\begin{tabular}{|c|ccc|}
\hline Ring radius, $\boldsymbol{\mu m}$ & $\mathbf{F S R}, \mathbf{n m}$ & $\begin{array}{c}\text { Average ER, } \mathbf{d B} \\
\begin{array}{c}\text { Average } \mathbf{Q} \text {-factor, } \\
\mathbf{d B}\end{array}\end{array}$ \\
\hline $\mathbf{1 5}$ & 9.8 & 16.0 & 355.5 \\
\hline $\mathbf{2 0}$ & 7.3 & 15.5 & 845.9 \\
\hline $\mathbf{2 5}$ & 5.8 & 7.1 & $1,281.1$ \\
\hline
\end{tabular}

In addition to their applications in optical filtering and signal processing, SWG ring resonators can be used for sensing applications. For example, by operating in the subwavelength regime, the evanescent field is enhanced which can be useful for sensing applications. Moreover, since the Bloch mode exists between the silicon blocks, index sensing is possible. With SWG ring resonators without a top oxide cladding (i.e., an air cladding), sensitivities exceeding $383 \mathrm{~nm} / \mathrm{RIU}$ in water and 270 $\mathrm{nm} / \mathrm{RIU}$ in air, which are greater than those obtained with rings resonators based on conventional silicon slot and strip waveguides, were demonstrated [15]. 


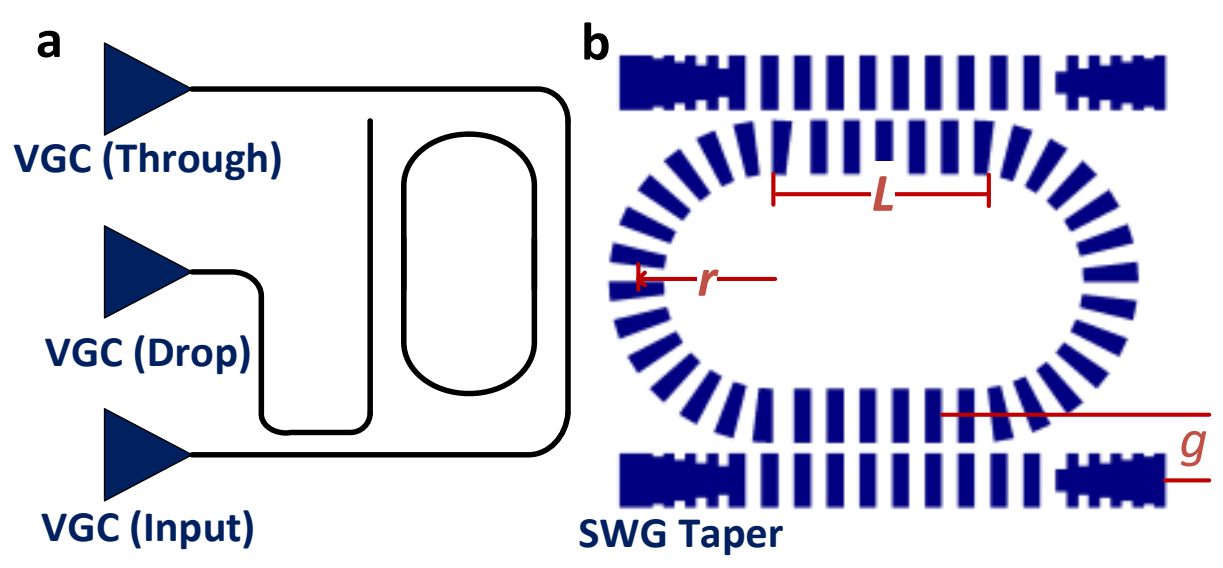

Fig. 9. SWG racetrack resonator: (a) schematic of device and (b) detailed view.

Racetrack resonators may be preferable as they allow for a better control over the coupling efficiency since the coupling region between the resonator and the bus waveguides is longer [36]. By controlling the coupling length, we can achieve different coupling conditions, i.e., under-coupled, critically coupled, and overcoupled. Figure 9 shows the layout and a zoom of an SWG racetrack resonator. The length of the directional coupling section is $L$ and the gap is denoted $g$, which are the two parameters that determine the coupling efficiency and operating condition (undercoupled, critically coupled, over-coupled) of the resonator. The curved section has a radius $r$ and the total perimeter of the racetrack which determines the FSR is $2 \pi r+$ $2 L$.

To demonstrate the design, we consider SWG waveguides with the same parameters as above and the racetrack resonator has $r=20 \mu \mathrm{m}, L=40 \mu \mathrm{m}$, and $g=$ $600 \mathrm{~nm}$. Figure 10 shows the measured through and drop responses of the SWG racetrack resonator (the typical fiber-to-fiber loss is $\sim 16 \mathrm{~dB}$ ). The measured FSR is $4.6 \mathrm{~nm}$ and with an ER as high as $33 \mathrm{~dB}$; the $3 \mathrm{~dB}$ bandwidth is $1 \mathrm{~nm}$. Compared to SWG ring resonators, the ER is higher such that the structure may be useful in the designs of SWG modulators. The design can be improved further by increasing the bending radius and optimizing the period of the SWG bus and ring waveguides to reduce losses further. Moreover, the gap can be adjusted to optimize the coupling (e.g., to improve the through and/or drop responses). 


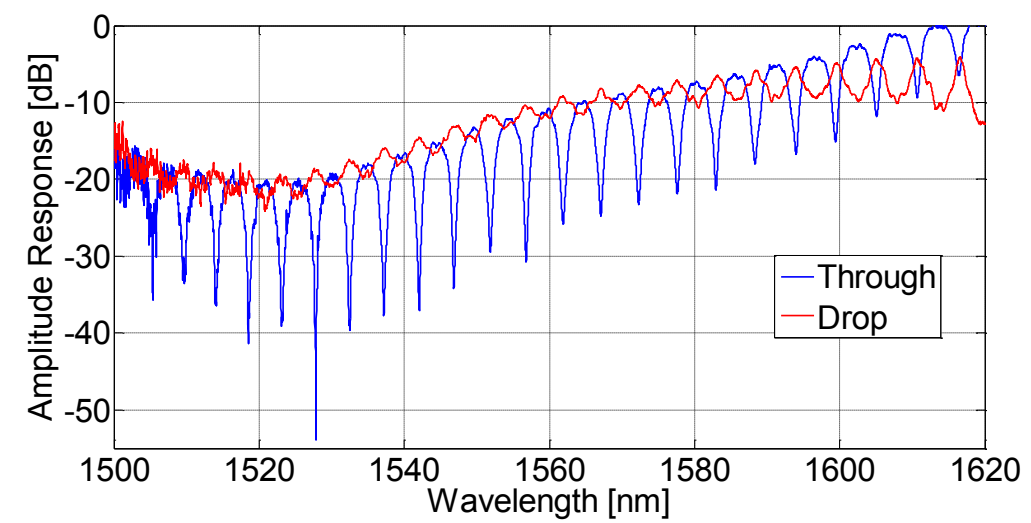

b

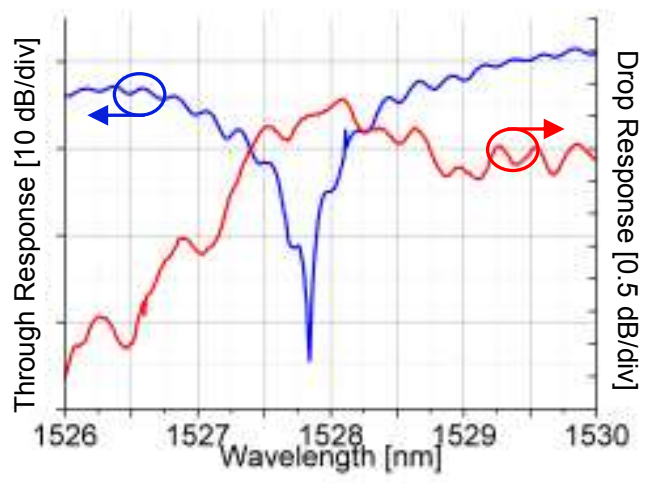

Fig. 10. (a) Measured through and drop responses of a SWG racetrack resonator and (b) zoom of the resonance at $1527.85 \mathrm{~nm}$. See text for device parameters.

\section{SWG delays}

ODLs are fundamental building blocks that enable many photonic signal processing functions in optical communications and microwave photonic systems. For example, ODLs can enable optical buffering, timing jitter control, packet synchronization, and time slot interchange, as well as be implemented in microwave filtering and phase shift control devices to process broadband $\mathrm{RF}$ signals in radio-over-fiber communications and radar systems [37,38]. Compared to all-fiber and micro-electromechanical-system (MEMS) based counterparts [39,40], integrated waveguide ODLs are more compact, provide greater temporal resolution, and support broader RF bandwidth $[34,35,41]$.

The two general ODL approaches to induce a time delay $(\Delta t)$ between optical pulses (signals) are based on variation of the propagation length $(L)$, referred to as a length-variable ODL, and variation of the propagation group velocity $\left(v_{\mathrm{g}}\right)$, i.e., $\Delta t=$ $\Delta\left(L / v_{\mathrm{g}}\right)$, referred to as wavelength-variable ODL. In the latter approach, a highly dispersive waveguide is used to create group velocity variation along different wavelengths. The pulses need to be carried on different optical wavelengths to experience different propagation velocities and correspondingly different propagation time delays. However, in applications where changing wavelengths in the optical pulses to obtain a change in time delay cannot be used, a length-variable approach must be adopted. For length-variable waveguide ODLs, the time delay between the waveguides (taps) of the delay line is obtained by changing the length of the waveguides in the waveguide array. In this design, the waveguide array implemented on chip requires a curvy or spiral topology which, regardless of the material platform, 
consumes a considerable portion of the chip area for creating the required time delay between waveguides.

The use of SWG to create a delay to implement a Mach-Zehnder interferometer (MZI) for all-optical switching applications has been demonstrated recently [42]. We build on this approach and propose an integrated waveguide ODL solution based on SWG waveguides which reduces the complexity of the device design by implementing the required incremental time delay difference through a precise change of the group index in each waveguide branch. We refer to this as an index-variable approach.

Figure 11(a) shows a schematic of our proof-of-principle device structure for inducing time delays between optical pulses. The ODL structure comprises a 4-arm MZI to implement 4 taps. Each tap has one SWG waveguide, each with the same length (in this case, $L=8 \mathrm{~mm}$ ). As discussed in Section 2, the duty cycle determines the group index of the SWG waveguide and hence, the properties of the propagating Bloch mode. By using a different duty cycle $D_{i}$ for each SWG branch, different optical paths among the waveguides all having the same length $(L)$ can be engineered to control the temporal separation between the taps.

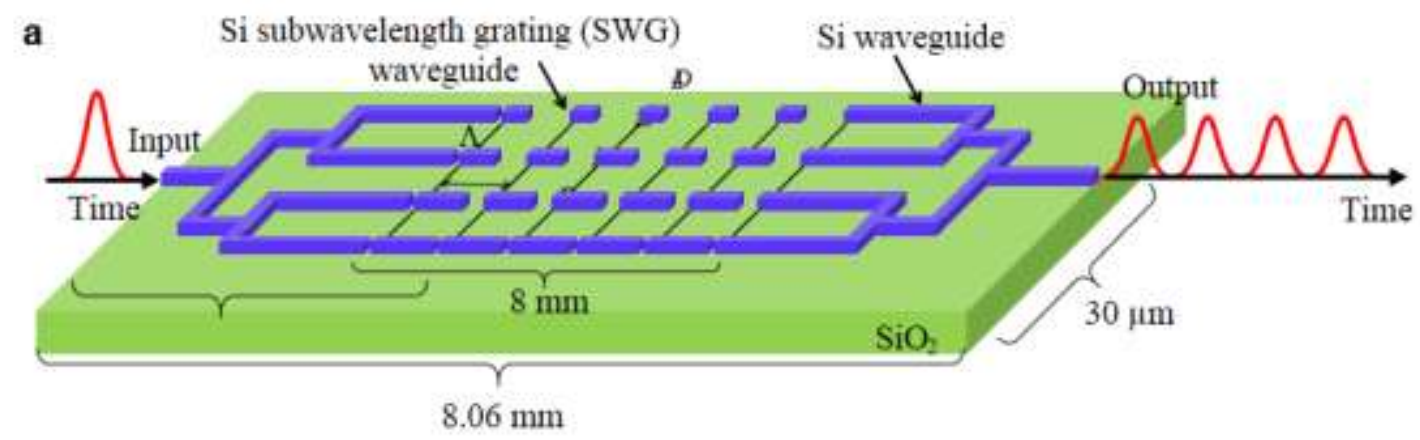

b

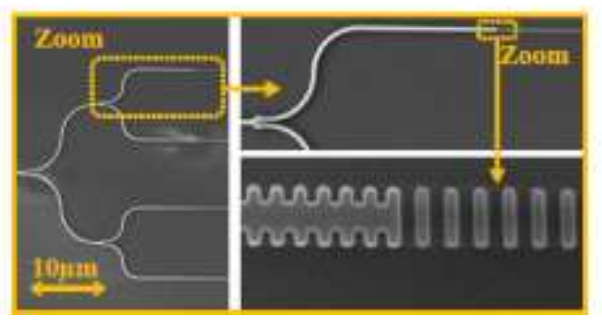

Fig. 11. (a) Schematic and (b) an SEM image of 4-tap ODL structure based on SWG with different duty cycles, $D_{1}=60 \%, D_{2}=50 \%, D_{3}=40 \%$, and $D_{4}=30 \%$.

The ODL structure is realized on silicon-on-insulator (SOI) in a chip area of $0.24 \mathrm{~mm}^{2}$, i.e., $30 \mu \mathrm{m}$ (width) $\times 8.06 \mathrm{~mm}$ (length). The $\mathrm{SWG}$ waveguides have a period $\Lambda=250 \mathrm{~nm}$ and a cross-section $W=500 \mathrm{~nm} \times h=220 \mathrm{~nm}$. This indexvariable ODL has been designed to have $\sim 10$ ps incremental time-step between the taps. The conventional length-variable ODL on the same SOI platform, had a chip area of $\sim 90 \mathrm{~mm}^{2}$, i.e., $4.5 \mathrm{~mm}$ (width) $\times 20 \mathrm{~mm}$ (length), while providing similar optical time delays ( $\sim 10 \mathrm{ps})$, by considering 4 taps of the structure. As before, SWG tapers and VGCs (optimized for TE transmission) are used. An SEM image taken before the deposition of the top oxide cladding layer is shown in Fig. 11(b). 
The duty cycles in the SWG waveguides are $D_{1}=60 \%, D_{2}=50 \%, D_{3}=40 \%$, and $D_{4}=30 \%$. Six Y-branches (with 50:50 splitting ratio) are used to split and collect the optical power at the input and output of the SWG waveguides, respectively. The measured loss of the ODL structure, including the loss of the Y-branches and the propagation loss through the SWG waveguides, is $\sim 16 \mathrm{~dB}$.

Figure 12(a) shows the measured spectral response of the fabricated ODL. Since the ODL is essentially a 4-arm interferometer, the spectral response corresponds to the interference fringes. The dominant spectrally periodic features have a period of $\sim 0.9 \mathrm{~nm}$, which corresponds to an incremental time delay of $\sim 9$ ps between adjacent taps, i.e., between pairs of adjacent waveguide arms forming the interferometer (this time delay difference corresponds to a group refractive index difference of 0.338 between adjacent SWG waveguides). Note that spectral periodic features with smaller periods can also be observed and these are associated with the existing larger time delays in the ODL (i.e., not between pairs of adjacent waveguide arms). We then launch a Gaussian-like optical pulse from a mode-locked laser with a full width at half maximum (FWHM) bandwidth of $2 \mathrm{~nm}$ at a central wavelength of $1556 \mathrm{~nm}$ into the ODL. Figure 12(b) shows the generated time-domain output pulse train measured using a $500 \mathrm{GHz}$ optical sampling oscilloscope. The generated time-delay difference between the taps of the OTTDL is $\sim 9 \mathrm{ps}$, which is in agreement with the spectral response measurements. Note that the pulse propagating through the SWG waveguide with the lowest duty cycle (i.e., $D_{4}$ ) arrives faster than the pulses in the other branches.
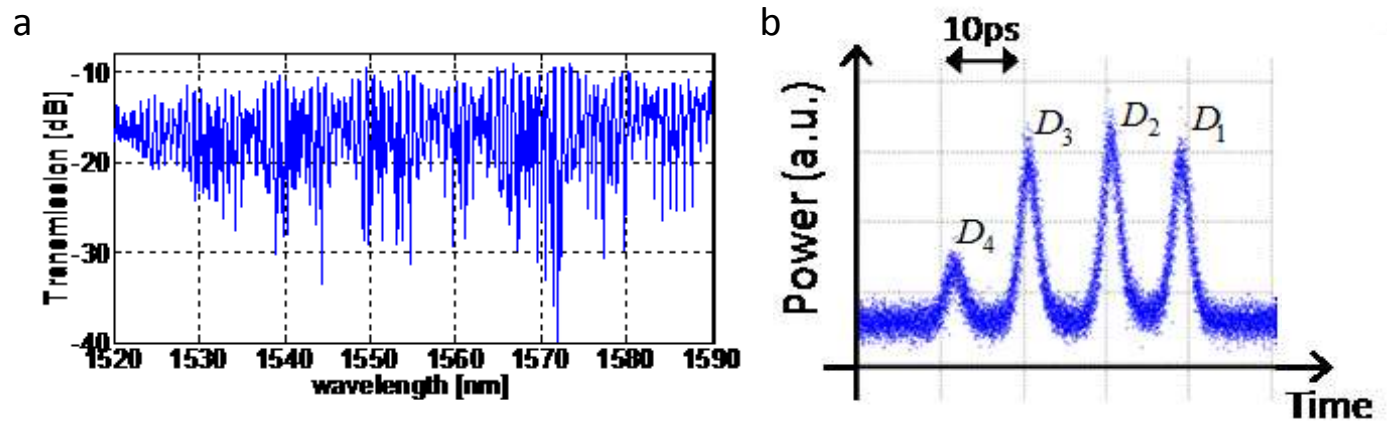

Fig. 12. (a) Measured spectral response of the fabricated 4-tap MZI ODL. (b) Generated time domain pulse train at the output of the fabricated ODL in response to a single input optical pulse.

This demonstration clearly show the potential of the proposed integrated waveguide ODL for realizing time delays of a few ps with $8 \mathrm{~mm}$ long SWG waveguides. Note that the minimum achievable time delay scales down proportionally with the SWG length, and femtosecond time delay resolutions can be readily achieved in shorter waveguides $(<8 \mathrm{~mm})$.

\section{Discussion and summary}

We have provided an overview of recent work on developing wavelength selective SWG waveguide filters based on BGs and ring resonators as well as index-variable ODLs. For SWG BGs, the peak reflection wavelength can be tuned by adjusting the duty cycle of the interleaved SWG waveguides. The fabricated SWG ring resonators exhibit Q-factors that are comparable to conventional ring resonators in SOI; higher 
Q-factors are possible by properly adjusting/optimizing the SWG waveguide parameters such as duty cycle and bend radius. The SWG waveguide structures considered in this paper all involve the use of an index-matched top oxide cladding. It is possible to use no top cladding, i.e., an air cladding, or to deposit a specific material such as a polymer for the top cladding. This gives flexibility in tailoring the optical properties of the SWG waveguide structures to be different from conventional SOI waveguides and provides new opportunities to implement tuning mechanisms or offers enhanced evanescent field interactions for sensing. Another main advantage of SWG waveguide structures lies in the possibility to achieve greater compactness, especially for racetrack ring resonator configurations which typically have higher ER; this in turn paves the way for coupled-resonator-optical waveguides (CROW)-based modulators [43]. We have also demonstrated a novel design to realize compact ODLs that can support very broad operation bandwidth and should serve as a fundamental step towards the development of many photonic integrated circuits to realize optical and microwave photonics signal processing functions. Note that we considered devices optimized for TE operation only; however, it is also possible to design and operate SWG waveguides and components with TM modes [9]. While better fabrication/processing is required to improve the spectral responses/properties of both the BGs (e.g., to reduce the broadband reflections) and ring resonators (e.g., to obtain greater $Q$-factor and ER), the structures and devices described in this paper will enable new functionalities that can be added into the SWG waveguide component toolbox.

\section{Acknowledgments}

This work was supported in part by the NSERC NGON and SiEPIC CREATE programs, NSERC SPG, and the Royal Society International Exchanges Scheme 2012/R2. The devices were fabricated by R. Bojko at the University of Washington Nanofabrication Facility, a member of the NSF National Nanotechnology Infrastructure Network. We thank Dr. R. Ashrafi (McGill) for his contributions to the development of the SWG ODLs.

\section{References}

[1] M. Lipson, "Silicon photonics: the optical spice rack," IET Electronics Letters, vol. 45, pp. 575577, 2009.

[2] R. Halir, A. Ortega-Moñux, J. H. Schmid, C. Alonso-Ramos, J. Lapointe, D.-X. Xu, J. G. Wangüemert-Pérez, I. Molina-Fernández, and S. Janz, "Recent advances in silicon waveguide devices using sub-wavelength gratings," IEEE Journal of Selected Topics in Quantum Electronics, vol. 20, pp. 279-291, 2014.

[3] R. Halir, P. J. Bock, P. Cheben, A. Ortega-Moñux, C. Alonso-Ramos, J. H. Schmid, J. Lapointe, D.-X. Xu, J. G. Wangüemert-Pérez, Í. Molina-Fernández, and S. Janz, "Waveguide subwavelength structures: a review of principles and applications," Lasers and Photonics Review, vol. 9, pp. 25-49, 2014.

[4] R. Halir, P. Cheben, S. Janz, D.-X. Xu, Í. Molina-Fernández, and J. G. Wangüemert-Pérez, "Waveguide grating coupler with subwavelength microstructures," Optics Letters, vol. 34, pp. 1408-1410, 2009.

[5] R. Halir, P. Cheben, J. H. Schmid, R. Ma, D. Bedard, S. Janz, D.-X. Xu, A. Densmore, J. Lapointe, and Í. Molina-Fernández, "Continuously apodized fiber-to-chip surface grating coupler with refractive index engineered subwavelength structure," Optics Letters, vol. 35, pp. 3243-3245, 2010.

[6] P. J. Bock, P. Cheben, J. H. Schmid, J. Lapointe, A. Delâge, S. Janz, G. C. Aers, D.-X. Xu, A. Densmore, and T. J. Hall, "Subwavelength grating periodic structures in silicon-on-insulator: a new type of microphotonic waveguide," Optics Express, vol. 18, pp. 20251-20262, 2010.

[7] A. Ortega-Moñux, L. Zavargo-Peche, A. Maese-Novo, Í. Molina-Fernández, R. Halir, J. Wangüemert-Pérez, P. Cheben, and J. H. Schmid, "High-performance multimode interference 
coupler in silicon waveguides with subwavelength structures," IEEE Photonics Technology Letters, vol. 23, pp. 1406-1408, 2011.

[8] P. J. Bock, P. Cheben, J. H. Schmid, J. Lapointe, A. Delâge, D.-X. Xu, S. Janz, A. Densmore, and T. J. Hall, "Subwavelength grating crossings for silicon wire waveguides," Optics Express, vol. 18, pp. 16146-16155, 2010.

[9] V. Donzella, A. Sherwali, J. Flueckiger, S. T. Fard, S. M. Grist, and L. Chrostowski, "Subwavelength grating components for integrated optics applications on SOI chips," Optics Express, vol. 22, pp. 21037-21050, 2014.

[10] P. J. Bock, P. Cheben, J. H. Schmid, A. Delâge, D.-X. Xu, S. Janz, and T. J. Hall, "Subwavelength grating mode transformers in silicon slab waveguides," Optics Express, vol. 17, pp. 19120-19133, 2009.

[11] A. V. Velasco, M. L. Calvo, P. Cheben, A. Ortega-Moñux, J. H. Schmid, C. A. Ramos, Í. Molina-Fernández, J. Lapointe, M. Vachon, S. Janz, and D.-X. Xu, "Ultracompact polarization converter with a dual subwavelength trench built in a silicon-on-insulator waveguide," Optics Letters, vol. 37, pp. 365-367, 2012.

[12] J. Wang, I. Glesk, and L. R. Chen, "Subwavelength grating filtering devices," Optics Express, vol. 22, pp. 15335-15345, 2014.

[13] J. Wang, I. Glesk, and L. R. Chen, "Subwavelength grating Bragg grating filters in silicon-oninsulator," IET Electronics Letters, vol. 51, pp. 712-713, 2015.

[14] J. Wang, I. Glesk, and L. R. Chen, "Subwavelength grating filters in SOI," in $12^{\text {th }}$ International Conference on Group IV Photonics, 2015.

[15] V. Donzella, A. Sherwali, J. Flueckiger, S. M. Grist, S. T. Fard, and L. Chrostowski, "Design and fabrication of SOI micro-ring resonators based on sub-wavelength grating waveguides," Optics Express, vol. 23, pp. 4791-4803, 2015.

[16] R. Ashrafi, J. Wang, I. Glesk, and L. R. Chen, "Silicon photonic subwavelength grating based integrated optical delay lines," in $12^{\text {th }}$ International Conference on Group IV Photonics, 2015.

[17] S. M. Rytov, "Electromagnetic properties of a finely stratified medium," Soviet Physics JETPUSSR, vol. 2, pp. 466-475, 1956.

[18] P. Lalanne and J. P. Hugonin, "High-order effective-medium theory of subwavelength gratings in classical mounting: application to volume holograms," Journal of the Optical Society of America A-Optics Image Science and Vision, vol. 15, pp. 1843-1851, 1998.

[19] R. Kashyap, Fiber Bragg Gratings. Academic Press, 1999.

[20] T. A. Strasser, T. Erdogan, A. E. White, V. Mizrahi, and P. J. Lemaire, "Ultraviolet laser fabrication of strong nearly polarisation-independent Bragg reflectors in germanium-doped silica waveguides on silica subtstrates," Applied Physics Letters, vol. 65, pp. 3308-3310, 1994.

[21] J. Albert, F. Bilodeau, D. C. Johnson, K. O. Hill, S. J. Mihailov, D. Stryckman, T. Kitagawa, and Y. Hibino, "Polarisation-independent strong Bragg gratings in planar lightwave circuits," IET Electronics Letters, vol. 34, pp. 485-486, 1998.

[22] T. E. Murphy, J. T. Hastings, and H. I. Smith, "Fabrication and characterization of narrow-band Bragg-reflection filters in silicon-on-insulator ridge waveguides," IEEE/OSA Journal of Lightwave Technology, vol. 19, pp. 1938-1942, 2001.

[23] X. Wang, W. Shi, H. Yun, S. Grist, N. A. F. Jaeger, and L. Chrostowski, "Narrow-band waveguide Bragg gratings on SOI wafers with CMOS-compatible fabrication process," Optics Express, vol. 20, pp. 15547-15558, 2012.

[24] G. E. Kohnke, C. H. Henry, E. J. Laskowski, M. A. Cappuzzo, T. A. Strasser, and A. E. White, "Silica based Mach-Zehnder add-drop filter fabricated with UV induced gratings," IET Electronics Letters, vol. 32, pp. 1579-1580, 1996.

[25] Y. Hibino, T. Kitagawa, K. O. Hill, F. Bilodeau, B. Malo, J. Albert, and D. C. Johnson, "Wavelength division multiplexer with photoinduced Bragg gratings fabricated in a planarlightwave-circuit-type a symmetric Mach-Zehnder interferometer on Si," IEEE Photonics Technology Letters, vol. 8, pp. 84-86, 1996.

[26] J. M. Jouanno, D. Zauner, and M. Kristensen, "Low crosstalk planar optical add-drop multiplexer fabricated with UV-induced Bragg gratings," IET Electronics Letters, vol. 33,pp. 2120-2121, 1997.

[27] M. Burla, L. R. Cortés, M. Li, X. Wang, L. Chrostowski, and J. Azaña, "Integrated waveguide Bragg gratings for microwave photonics signal processing," Optics Express, vol. 21, pp. 2512025147, 2013.

[28] J. Wang, R. Ashrafi, M. Rochette, and L. R. Chen, "Chirped microwave pulse generation using an integrated SiP Bragg grating in a Sagnac loop," IEEE Photonics technology Letters, vol. 27, pp. $1876-1879,2015$. 
[29] J. Čtyroký, P. Kwiecien, J. Wang, I. Richter, I. Glesk, and L. R. Chen, "Simulations of waveguide Bragg grating filters based on subwavelength grating waveguide," in SPIE Optics+ Optoelectronics, 2015, pp. 95160M-95160M-7.

[30] Y. Zhang, S. Yang, A. E.-J. Lim, G.-Q. Lo, C. Galland, T. Baehr-Jones, and M. Hochberg, "A compact and low loss Y-junction for submicron silicon waveguide," Optics Express, vol. 21, pp. 1310-1316, 2013.

[31] Y. Wang, X. Wang, J. Flueckiger, H. Yun, W. Shi, R. Bojko, N A. F. Jaeger, and L. Chrostowski, "Focusing sub-wavelength grating couplers with low back reflections for rapid prototyping of silicon photonic circuits," Optics Express, vol. 22, pp. 20652-20662, 2014.

[32] S. J. Xiao, M. H. Khan, H. Shen, and M. H. Qi, "A highly compact third-order silicon microring add-drop filter with a very large free spectral range, a flat passband and a low delay dispersion," Optics Express, vol. 15, pp. 14765-14771, 2007.

[33] Q. F. Xu, B. Schmidt, S. Pradhan, and M. Lipson, "Micrometre-scale silicon electro-optic modulator," Nature, vol. 435, pp. 325-327, 2005.

[34] F. F. Liu, Q. Li, Z. Y. Zhang, M. Qiu, and Y. K. Su, "Optically tunable delay line in silicon microring resonator based on thermal nonlinear effect," IEEE Journal of Selected Topics in Quantum Electronics, vol. 14, pp. 706-712, 2008.

[35] J. Cardenas, M. A. Foster, N. Sherwood-Droz, C. B. Poitras, H. L. R. Lira, B. B. Zhang, A. L. Gaeta, J. B. Khurgin, P. Morton, and M. Lipson, "Wide-bandwidth continuously tunable optical delay line using silicon microring resonators," Optics Express, vol. 18, pp. 26525-26534, 2010.

[36] W. Bogaerts, P. De Heyn, T. Van Vaerenbergh, K. De Vos, S. K. Selvaraja, T. Claes, P. Dumon, P. Bienstman, D. Van Thourhout, and R. Baets, "Silicon microring resonators," Laser \& Photonics Reviews, vol. 6, pp. 47-73, 2012.

[37] A. E. Willner, B. Zhang, L. Zhang, L. Yan, and I. Fazal, "Optical signal processing using tunable delay elements based on slow light," IEEE Journal of Selected Topics in Quantum Electronics, vol. 14, pp. 691-705, 2008.

[38] X. H. Zou, W. Pan, and L. S. Yan, "All-optical processing to optical and radio frequency (RF) signals," Science Bulletin, vol. 60, pp. 2151-2153, 2015.

[39] W. Ng, A. A. Walston, G. L. Tangonan, J. J. Lee, I. L. Newberg, and N. Bernstein, "The first demonstration of an optically steered microwave phased array antenna using true-time-delay," IEEE/OSA Journal of Lightwave Technology, vol. 9, pp. 1124-1131, 1991.

[40] S. Jong-Dug, L. Back-Song, and K. Boo-Gyoun, "Optical true time-delay feeder for X-band phased array antennas composed of $2 \times 2$ optical MEMS switches and fiber delay lines," IEEE Photonics Technology Letters, vol. 16, pp. 1364-1366, 2004.

[41] H. Lee, T. Chen, J. Li, O. Painter, and K. J. Vahala, "Ultra-low-loss optical delay line on a silicon chip," Nature Communications, vol. 3, p. 867, 2012.

[42] I. Glesk, P. J. Bock, P. Cheben, J. H. Schmid, J. Lapointe, and S. Janz, "All-optical switching using nonlinear subwavelength Mach-Zehnder on silicon," Optics Express, vol. 19, pp. 1403114039, 2011.

[43] J. K. S. Poon, J. Scheuer, Y. Xu, and A. Yariv, "Designing coupled-resonator optical waveguide delay lines," Journal of the Optical Society of America B-Optical Physics, vol. 21, pp. 1665$1673,2004$. 